\title{
Concepciones de violencia intrafamiliar de los militares del Batallón de Comunicaciones del Ejercito Nacional a partir de sus configuraciones familiares
}

Domestic violence notions of military of the National Army

Communications Batallion from their family structure

Autores: Ortiz Quevedo Jenny Patricia, Bravo Dedfor Crisanto, Arismendi

Mazabel Robert

DOI: https://doi.org/10.25058/1794600X.1799 


\title{
CONCEPCIONES DE VIOLENCIA INTRAFAMILIAR DE LOS MILITARES DEL BATALLÓN DE COMUNICACIONES DEL EJERCITO NACIONAL A PARTIR DE SUS CONFIGURACIONES FAMILIARES*
}

\author{
Domestic violence notions of military of the National Army \\ Communications Batallion from their family structure
}

Concepções de violência intrafamiliar por militares do Batalhão de Comunicações do Exército Nacional a partir de suas configurações familiares

\author{
Ortiz Quevedo Jenny Patricia ${ }^{a}$ \\ sicojenny10@gmail.com \\ Bravo Dedfor Crisanto ${ }^{b}$ \\ dedfor@hotmail.co \\ Arismendi Mazabel Robert ${ }^{c}$ \\ robert.arismendy@gmail.com
}

Fecha de recepción: 17 de abril de 2020 Fecha de revisión: 21 de abril de 2020 Fecha de aceptación: 04 de mayo de 2020

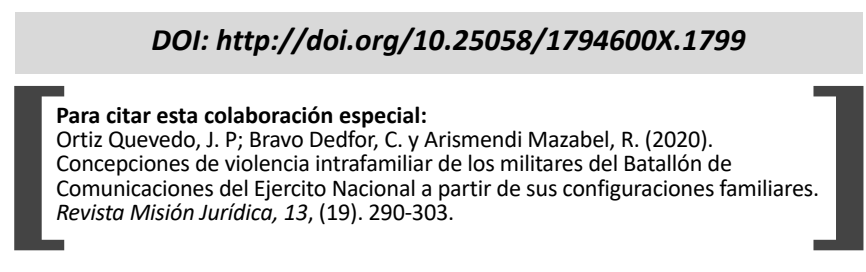

\section{RESUMEN}

La violencia intrafamiliar es un tema que impacta a la sociedad en general, la forma en la que es concebida y las relaciones que de allí se desprenden juegan un papel fundamental en la cotidianidad de los miembros del grupo familiar, en este sentido, el estudio se propuso comprender las concepciones de Violencia Intrafamiliar a partir de las configuraciones familiares que tiene un grupo de soldados, suboficiales y oficiales pertenecientes al Batallón de Comunicaciones del Ejercito Nacional de Colombia. Para tal fin, se utilizó el enfoque cualitativo indagando de forma directa mediante dos técnicas complementarias, el grupo focal y el cuestionario, de acuerdo con los presupuestos metodológicos de Bonilla-Castro y Rodríguez. Los resultados arrojaron que la concepción de violencia intrafamiliar por parte de los entrevistados es entendida como todo daño físico, emocional y social contra los familiares, dentro de las condiciones que promueven la misma se manifiestan, las expresiones irrespetuosas, falta de escucha activa, el distanciamiento personal y fallas en la comunicación asertiva debido a falencias en la regulación emocional y el

\footnotetext{
*Artículo de Reflexión.

a. Máster en Educación. Universidad Santo Tomas. Especialista en pedagogía y docencia Universitaria. Universidad San buenaventura. Psicóloga. Universidad Iberoamericana.

b. Magíster en Educación. Universidad Santo Tomas, Profesional en Ciencias militares. Escuela Militar de Cadetes General José María Córdoba. Administrador de Empresas. Universidad Militar Nueva Granada.

c. Doctorado en Neurociencia desarrollo y educación. Universidad de Sonora. . Máster en ciencias de la familia. Universidad Santiago de Compostela. Especialista en docencia. Universitaria. Universidad Iberoamericana. Ciencias de la Educación Universidad de la Salle.
} 
nivel de formación de la pareja. Se concluye que la violencia puede estar asociada a la configuración familiar que han constituido, siendo los padres quienes recurren con mayor frecuencia a ella, estas emergen de forma inconsciente debido a las pautas de crianza y las experiencias en su infancia.

\section{PALABRAS CLAVES}

Familia, configuraciones familiares, Violencia intrafamiliar, tipos de violencia.

\section{ABSTRACT}

Domestic violence is an issue that impacts society at large, the way in which it is conceived and the relationships that are derived from it play a key role in the everyday life of the family group, in this sense, this research has the purpose of understanding the noions of domestic violence from the family structure that a group of soldiers, non-comissioned and comissioned officers of the National Army Communications Batallion have. To this end, a qualitative approach was used, making an direct examination with two complementary techniques, the focus group and the questionnaire, in accordance with the methodological assumptions of Bonilla-Castro and Rodríguez.

The results indicated that the concept of domestic violence by the officers interviewed is understood as all physical, emotional and social damage against family members, within the conditions that promote it are manifested the disrispectful expressions, the lack of active listening, personal distance and flaws in assertive communication due to failures in emotional regulation and the couple's level of training. It is concluded that violence may be associated to the family structure that parents have constituted, being the ones that more frequently recur to it, this emerge in an uncoscious way because of their parenting patterns and childhood experiencies.

\section{KEY WORDS}

Family, family structure, domestic violence, types of violence.

\section{RESUMO}

A violência intra familiar é um tema que impacta a sociedade em geral, a forma como é concebida e as relações que daí decorrem desempenham um papel fundamental no cotidiano dos membros do grupo familiar, neste sentido, no estudo se propôs compreender as concepções de violência intrafamiliar a partir das configurações familiares de um grupo de soldados, sargentos e oficiais do Batalhão de Comunicações do Exército Nacional da Colômbia. Para tanto, utilizou-se a abordagem qualitativa, investigando diretamente por meio de duas técnicas complementares, o grupo focal e o questionário, de acordo com os pressupostos metodológicos de Bonilla-Castro e Rodríguez.

Os resultados mostraram que a concepção de violência intrafamiliar pelos entrevistados é entendida como todo dano físico, emocional e social contra familiares, nas condições que o promovem, expressões desrespeitosas, falta de escuta ativa, distanciamento falhas de comunicação pessoal e assertiva devido a deficiências na regulação emocional e no nível de treinamento do parceiro. Conclui-se que a violência pode estar associada à configuração familiar que eles constituíram, sendo os pais que a ela recorrem com maior frequência, estes surgem inconscientemente devido aos padrões de educação e vivências na infância.

\section{PALAVRAS-CHAVE}

Família; configurações familiares; violência doméstica; tipos de violência.

\section{INTRODUCCIÓN}

La violencia intrafamiliar (en adelante VIF), genera impactos a nivel psicosocial, político y económico, alimentando diferentes problemáticas sociales, en este sentido, su incremento conlleva a la preocupación y reflexión de la sociedad en general, la no naturalización de esta propicia una mayor búsqueda de alternativas para retirarla de la cotidianidad de las familias. Por ejemplo, en el año 2019 según el Subsistema de Vigilancia de violencia intrafamiliar, maltrato infantil y violencia sexual (SIVIM), se presentaron en Colombia 397 casos por cada 100.000 habitantes, lo que implica que se debe revisar la comprensión frente al tema, ya que como lo afirma Valle (2012), las concepciones son un conjunto de conceptos esenciales que sustentan el análisis de objetos o fenómenos de estudio. En razón a lo anterior, es indispensable entender la violencia intrafamiliar 
en el marco de las configuraciones de sus familias, llevando al análisis y posteriormente generar la posibilidad de nuevas estrategias que aborden la situación.

Asimismo, se hace necesario describir el concepto de familia que según Rodrigo, Máiquez Chaves, Martín Quintana y Byme (2008), es un sistema dinámico de vínculos recíprocos expuesto a diversos cambios donde interactúan múltiples dimensiones del ser humano que propician factores protectores $\mathrm{y} / \mathrm{o}$ de riesgo. En razón a lo anterior, la configuración de la familia desde Carbonell, Carbonell y González (2012), es un elemento esencial en la vida de las personas, aún más en el ámbito militar, donde se construyen en su mayoría prácticas heterónomas aunadas a elevados niveles de estrés, además de las condiciones propias de trabajo en las bases militares que no les permiten estar continuamente con sus familias. Por tanto, se determinan las siguientes preguntas orientadoras ¿Cuáles son las concepciones de Violencia Intrafamiliar que tienen un grupo de soldados, suboficiales y oficiales pertenecientes a la Escuela de Comunicaciones del Ejercito Nacional de Colombia? y ¿Cómo estas concepciones de VIF inciden en la configuración familiar?

Es el caso de la Dirección de Familia y Bienestar del Ejército Nacional es claro el interés por el bienestar de las familias colombianas ya que en su misión se hace visible el cuidado del otro, siendo un pilar relevante en su estructura cognitiva social y esto también se proyecta a la familia de cada uno de sus miembros.

En esa misma línea, es imprescindible tener especial atención en las condiciones y particularidades de esta población, puesto que las situaciones como traslados a sitios lejanos, relaciones de pareja sostenidas en la distancia, escaso tiempo para compartir en familia, son determinantes en la configuración de los lazos familiares, como lo mencionan Páez, Mesa et al (2016), la familia es altamente influyente en relación a los comportamientos que tenga cada uno de sus miembros, en este sentido, se requiere escuchar estas voces en un tema de impacto social como es la violencia intrafamiliar.

Por otra parte, la investigación de Molina (2015), denominada "Vulnerabilidad y daño psiquico en mujeres victimas de violencia en el medio famliar", de orden cualitativo, señala las caracteristicas de la victimas y de la situacion de maltrato que se asocia a un daño psicofisico, siendo notorio el impacto negativo que tiene la exposicion a la violencia, tambien, la investigacion titulada la prevención del suicidio en las fuerzas militares de Colombia de la Universidad del Rosario, resalta que los Militares Jóvenes son los más vulnerables y más propensos al suicidio por la presión ejercida de la sociedad y su familia, situación que se debe revisar ya que las personas que trabajan en las Fuerzas Militares se exponen a cambios bruscos y a cambios constantes para sí mismo y sus familias lo que puede repercutir en su dinámica familiar.

En otra investigación, realizada al interior de la institución con las familias de los militares en "intervención de trabajo social con familia en las Fuerzas Militares" (HOSMIC, 2013). Con enfoque cualitativo, concluye que las esposas de los militares son consideradas mujeres sumisas trasladando sus propias metas a un lugar secundario. Por otra parte, (Arena \& otros,2013) en el proceso investigativo denominado "experiencia de la conyugalidad en los oficiales y suboficiales de la Fuerza Aérea Colombiana", se mencionan que las relaciones emocionales y los factores asociados a las uniones matrimoniales, tienen implicación en las interacciones entre sus miembros y marcan una dinámica particular.

A partir de lo anterior, es evidente la importancia del tema de la violencia intrafamiliar y la configuración de familias de los militares, ya que no es solo una población con grandes responsabilidades, los cuales afrontan retos en su labor diaria, aparte de ello, deben cuidar a sus propias familias y dar respuesta a las diversas situaciones en las que se sumerge el entorno familiar, en fin, más allá de la concepción se encuentra el ejercicio de la reflexión.

\section{LA FAMILIA Y SUS CONFIGURACIONES}

La familia ejerce un proceso formativo en sí misma y propicia las condiciones de desarrollo del ser humano, por tanto, la forma en la que se configura genera impactos en los elementos psicosociales de sus integrantes. González, Gross y Pulido (2014), afirman que la familia es el primer escenario de socialización donde se construyen valores, normas, se tejen vínculos afectivos $\mathrm{y}$ desempeños cognoscitivos, dicho esto, se entiende que la configuración familiar orienta las conductas 
de sus miembros, compuesto entre dinámicas endógenas y exógenas, reflejadas en las practicas socioculturales, psicoafectivas y ético-políticas, siendo de naturaleza cambiante y adaptativa con relación a las demandas del contexto.

En esta línea Carbonel (2012), citado por Ramos \& González (2017), concibe la familia como: "El lugar primordial donde se comparten y gestionan los riesgos sociales de sus miembros" (2017: 4); es decir, se desprenden los principios básicos de convivencia e interacción en la sociedad, sin olvidar la socialización secundaria a la que se exponen las personas, lo que conlleva a la apuesta por un proyecto de vida en común. En razón a lo anterior, es fundamental estudiar las relaciones familiares como unidades que integran el sistema relacional, ya que en el núcleo familiar se pueden generar desintegraciones, concebidas como la ausencia psicológica y física temporal, parcial o total de uno de los padres que generan repercusiones en los hijos, donde la familia inicia con un proceso de descomposición o situaciones problémicas al interior de la misma (Básmeson \& Caicedo, 2011).

Las familias se configuran de diversas maneras, siendo las relaciones familiares las que permean la seguridad de sus miembros y el desarrollo psicológico, social, cultural y económico, así como las conductas individuales y grupales que, a su vez, determinan la cohesión, adaptación y establecen factores protectores o de riesgo frente a la VIF. Autores como Guío (2009) y Vila (2008), expresan que la familia evoca relaciones de parentesco y vínculos afectivos, por tanto, los integrantes del grupo familiar asumen la diversidad de roles que permean la conducta al interior y exterior de su medio.

Ahora bien, a partir de las configuraciones familiares, se desarrolla la convivencia familiar desplegando una serie de condiciones que fomentan diversas dinámicas en el núcleo familiar, impactando a cada individuo debido a que el comportamiento individual incide en las conductas de grupo, Stevenson y Baker (Citado por Espitia y Montes 2009), explican que el nivel de funcionalidad familiar se estructura a partir de las relaciones vinculantes frente a las dinámicas contextuales, siendo un reto sostener las bases de la configuración familiar.

\section{UNA MIRADA A LA VIOLENCIA INTRAFAMILIAR}

A partir de los años 70 se profundiza en la violencia intrafamiliar, como afirma Alonso \& Castellanos (2006), es en esta época donde la preocupación por la violencia contra la mujer cobra fuerza no solo en Colombia sino a nivel internacional, como resultado, en 1979 se firma la Convención sobre la eliminación de todas las formas de discriminación contra la Mujer, también conocida por sus siglas en inglés (CEDAW), esta, entre muchos otros lineamientos, ha marcado un camino de sensibilización en torno al tema y de proactividad de los entes que se encargan de la protección de las personas y su familia, no obstante, en la actualidad la presencia de VIF en los hogares es alta. En este sentido, en los meses de enero y mayo de 2018 se presentaron 32.445 casos de violencia intrafamiliar en Colombia, lo que implica una búsqueda de soluciones que debe emerger de una sociedad consciente y de un Gobierno Nacional y local responsable y articulado.

Cabe recordar que el marco legal del tema está conformado por los postulados internacionales de derechos humanos, la Constitución Política de 1991 y las leyes colombianas relacionadas con la familia. Ha de entenderse que la Violencia Intrafamiliar se contempló inicialmente según el Código Penal Colombiano (Ley 599 del 2000), en el artículo 229 de la siguiente manera "El que maltrate física o psicológicamente a cualquier miembro de su núcleo familiar, incurrirá, siempre que la conducta no constituya delito sancionado con pena mayor, en prisión de cuatro (4) a ocho (8) años" (2000.p.190), sin embargo, para que este fenómeno fuese considerado legal y penalmente como un delito, fue necesario en primera instancia lograr la aceptación de la familia como "núcleo fundamental de la sociedad" (artículo 42 de la Constitución de 1991).

Al hacer referencia a la violencia, no es exclusivamente aquella que se ejerce contra la mujer, en primera instancia se entiende como el uso intencional de la fuerza física, las amenazas contra uno mismo u otra persona, un grupo o una comunidad; en consecuencia, es muy probable que generen traumatismos, daños psicológicos, problemas de desarrollo o incluso la muerte (OMS, 2013). Es decir, el término violencia, puede referirse a los procesos (Navarro, 2013) donde coexiste una víctima y un victimario que atenta 
contra la dimensión física, psíquica, emocional o sexual de una persona, amenazando su bienestar.

Acorde a lo anterior, la VIF según la Secretaria de Salud de Bogotá (2010), incluye el maltrato o la violencia cometida a cualquiera de sus miembros, en condiciones como el abandono, negligencia o descuido y el abuso sexual, siendo evidente que esta es una problemática de atención mundial y ha mostrado una prevalencia histórica sobre la mujer. Asimismo, la Organización Mundial de la Salud (2002), afirma que "la violencia es un importante problema de salud pública en todo el mundo" (2002: 6), y en 2013 emite un informe en el que se visibiliza la violencia conyugal y en la violencia sexual infligida por otra persona distinta de la pareja.

También, el Instituto Nacional de Medicina Legal y Ciencias Forenses (2016), registró 26.473 eventos de violencia ocurridos en el contexto familiar, sin incluir la violencia de pareja; siendo el $38,08 \%$ de las víctimas niños, niñas y adolescentes (NNA), 6,24 \% personas mayores y el 55,67\% otros familiares (2016: 211). Cifras que invitan a reflexionar en torno a las concepciones que se tienen a nivel social de VIF debido que a partir del pensamiento se genera el actuar de las personas.

En este sentido, el informe Forensis Datos para la vida (2016), precisa que una de las creencias a superar es la violencia de género ya que el maltrato se extiende a sus hijos e hijas, generando un círculo vicioso donde la conducta agresiva se naturaliza En este orden de ideas, las consecuencias de la violencia no son para la victima directa exclusivamente, por el contrario, repercute en la dinámica familiar y los efectos nocivos se hacen evidentes e impactan a la sociedad.

Para el año 2017, se registra un aumento significativo; entre enero y noviembre de ese año, según el informe "lesiones fatales y no fatales de causa externa según la clasificación del Contexto de Violencia y Desaparecidos", ocurrieron 70.806 casos de violencia intrafamiliar en el país (INMLCF, 2017). Cifras que son alarmantes visibilizando que en promedio se generan 210 casos al día a los que, además, se suman a las cifras de violencia sexual, psicológica y de género.

Se puede afirmar que si bien la violencia no es exclusiva de un sólo género o se ejerce por el rol que se desempeña en la familia, sí se resalta el hecho que las cifras evidencian una población vulnerable, ubicando a las mujeres, niños/as y adolescentes(NNA) en esa primera línea, lo cual afirma Guío (2009) y Vila (2008), repercute en una posible replica de patrones que afectan el desarrollo y bienestar social de los miembros de la familia y que genera un impacto en el contexto que viven las víctimas (Díaz Colorado, 2012).

Cabe señalar, que la familia como fundadora de comprensión, autoestima y seguridad personal, define en buena medida el proyecto de vida de sus miembros. Así pues, es preciso entender el papel que cumple esta dentro del contexto militar y contrastarlo con la realidad del país frente a la VIF, para lo cual es primordial detallar sus interpretaciones frente a este fenómeno.

Según el Ministerio de Salud y Protección Social (2012), la VIF se define como toda acción que busque causar daño a la integridad física, psicológica o incluso a la libertad de algún miembro de la familia, así mismo, relaciona la deprivación emocional como parte de esta violencia, es decir, desde el abandono hasta el acto violento generan una alta carga emocional, en consecuencia, sus efectos perduran en el tiempo para las personas que hayan vivido esta experiencia.

Síntomas como el estrés, la ansiedad, depresión y sin fines de patologías pueden generarse ante la exposición de la VIF, donde el sujeto se traslada a un mundo violento en el que la salida tendrá que enmarcarse en un desarrollo profundo y consciente de la inteligencia emocional y social, en muchos casos apoyo terapéutico y activación de redes sociales.

Asimismo, Pérez (2005), menciona que cuando se trata de VIF no puede generalizarse, ya que el fenómeno al estar en el ámbito privado de la familia, dificulta ubicar los factores, sobre todo en entornos que promueven condiciones de vulnerabilidad, lo que conlleva a la reflexión y la actuación ante estos elementos, los detonantes como sus efectos no son siempre los mismos, las características personales de sus miembros y las concepciones que se tienen de la VIF, tendrán un impacto en la cotidianidad.

Es relevante mencionar que las dinámicas familiares van a afectar la paz interior y por efecto 
la construcción de las personas que se encuentran inmersas en este escenario, allí se disminuirá la autonomía como lo afirman Mahecha, Ortiz \& Palacios(2018), siendo esta un factor relevante del desempeño de las personas en el contexto educativo, no obstante, es complejo adquirir las bases emocionales como la seguridad o autoconfianza, con la exposición a situaciones de violencia cualquiera que estas sean.

Teniendo en cuenta a Piza (2014), la importancia de indagar sobre la VIF no sólo radica en su alta incidencia, sino también en "la poca investigación y el bajo desarrollo literario sobre el tratamiento de salud física y mental" (2014: 5). Lo anterior resulta iluminador ante el panorama de la presencia de este fenómeno en el contexto colombiano, puesto que muchas veces su desconocimiento contribuye al mantenimiento y expansión del fenómeno.

Continuando con el tema, se observan algunos esfuerzos del gobierno colombiano a nivel legal mediante normativas y aumento de penas asociados a delitos de violencia de género y la implementación de estrategias en torno a la VIF, como porgramas de promocion e intervención social, sin embargo, aún no son suficientes para eliminar este fenómeno, puesto que los porcentajes de violencia intrafamiliar disminuyen en algunas zonas, aumentan en otras o se sostienen, de acuerdo con las cifras anuales presentadas por Instituto de Medicina Legal (2017).

En este sentido, Ocampo y Amar (2017), expone la violencia intrafamiliar como un problema de salud pública en el que están presentes factores académicos de los padres de familia, estado civil, condiciones socioeconómicas, prácticas de crianzas, aspectos socioculturales, políticos y factores contextuales. Cabe resaltar que para definir un caso de violencia familiar la relación debe ser crónica, permanente o periódica. Para esto, Garrido (2013), señala que esta se caracteriza a grandes rasgos por:

Una especie de legitimidad, donde es aceptada para resolver conflictos.

Asociación de una idea errada de violencia como práctica para el ejercicio de poder.

Relaciones psicoafectivas que afectan la conducta.
La familia, según González et al (2018), continúa siendo permeada por la violencia en Colombia, la red familiar es fundamental para la prospectiva del entorno, por tanto, la violencia intrafamiliar dependiendo del exceso o abuso de autoridad se puede categorizar en: física, psicológica, sexual etc. Por consiguiente, la VIF según Garrido (2013), se establece de forma cíclica y progresiva, tomando cada vez mayor fuerza, Por ejemplo para Martinez (2016), la violencia verbal y emocional conllevan a la desintegracion de los elementos escenciales del autoestima de la persona ya que las palabras al igual que los actos denigran tambien se presenta la violencia sexual como una acción agresiva en el que se obliga al contacto sexual.

Dentro de esta clasificación se encuentra la violencia económica definida como el control y abuso absoluto del poder financiero en el hogar, allí se propician castigos monetarios por parte del agresor, finalmente, se encuentra la violencia doméstica la cual se da normalmente de hombres a mujeres, de acuerdo con su estatus económico o por ser quien aporta monetariamente al hogar.

Por lo tanto, es obligación constitucional del Estado trabajar con los agentes sociales en el diseño, elaboración, ejecución y seguimiento de las políticas, estrategias, proyectos $\mathrm{y}$ acciones que pongan en movimiento las políticas sociales y los planes de desarrollo, la VIF no se puede naturalizar y es a partir de la visibilización que se propician los procesos reflexivos que conllevan al cambio de las dinámicas familiares, el silencio será entonces uno de los peores enemigos de las personas que se encuentran inmersos en la violencia.

\section{METODOLOGÍA}

Con la finalidad de comprender cómo entienden las concepciones de Violencia Intrafamiliar un grupo de soldados, suboficiales y oficiales pertenecientes al Batallón de Comunicaciones del Ejercito Nacional y la incidencia en la configuración familiar, se recurrió al enfoque de investigación cualitativo, que según Bonilla-Castro y Rodríguez (2015), promueve el interés por ingresar a la realidad social por parte de los involucrados, por tanto, coincide con los objetivos investigativos, precisamente, estas autoras guiaron el abordaje metodológico que se presenta a continuación: 
En el marco de esta orientación metodológica se desarrolla la investigación cumpliendo con la delimitación de la situación problema, posteriormente el trabajo de campo y por último se genera el análisis y conclusiones del proyecto.

\section{Configuración de la muestra}

El tipo de muestreo que se llevó a cabo fue intencional o selectivo el cual hace referencia a una decisión asumida al comienzo de la investigación de trabajar con una muestra de 15 integrantes

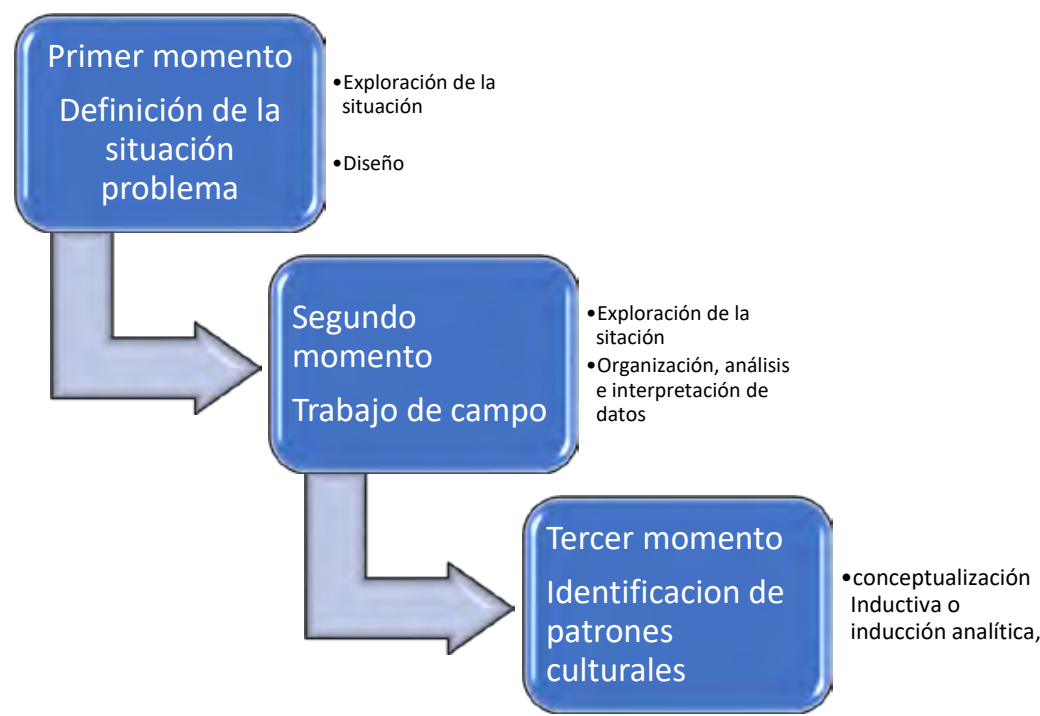

Gráfico 1. Momentos del proceso investigativo según Bonilla-Castro y Rodríguez (2015)

Elaboración propia

pertenecientes a la Batallón de Comunicaciones del Ejercito Nacional (5 soldados, 5 suboficiales y 5 oficiales), los cuales tienen edades entre los 30 a 40 años, con un tiempo de servicio en las Fuerzas Armadas entre 10 y 18 años, teniendo en cuenta que era un requisito tener un periodo amplio de tiempo con la experiencia de convivir en familia perteneciendo a la institución militar.

\section{Instrumentos}

El instrumento utilizado fue creado por el grupo investigador con la validación de seis investigadores en Ciencias Sociales y Humanas de las Universidades Santo Tomas, Fundación Universitaria San Alfonso y Universidad Colegio Mayor de Cundinamarca, a partir de dos categorías: familia y violencia intrafamiliar. En este se desglosan las subcategorías que dieron la línea a la estructuración de un cuestionario con doce preguntas, el cual fue aplicado en el grupo focal, debido a que esta técnica, como lo afirma Gurdián (2010), permite desde la experiencia personal comprender y reflexionar en torno a un fenómeno social de análisis que surgen de la problemática y la teorización, por lo que la información recolectada emergió de la grabación y posterior trascripción del grupo focal así como de la acomodación categorial del cuestionario, esto posibilitó el análisis categorial y presentación de las conclusiones del estudio.

\section{RESULTADOS}

Los resultados están expresados para su mayor comprensión en dos categorías (i) Familia y (ii) las concepciones de la VIF. Estas se determinan a partir de lo expresado por la población objeto de estudio en los grupos focales y la aplicación del cuestionario.

\section{Familia y sus configuraciones}

En los grupos se pudo determinar que el $90 \%$ de la muestra del Batallón de comunicaciones, concibe a la familia como la base de la 
sociedad, también para la conformación de sus grupos familiares, en primera instancia, son fundamentales los vínculos consanguíneos, aunque reconocen la importancia de los vínculos afectivos de los miembros para su afianzamiento. A este respecto en la aplicación del cuestionario en los grupos focales, el $10 \%$ de la población estudiada refiere una composición familiar desde la perspectiva legal, ya sea establecido desde el matrimonio civil o por rito religioso, siendo para este porcentaje de la población un pilar fundamental para la conformación de familia.

En cuanto a la composición, se halló que el $80 \%$ tiene un hogar de tipo nuclear, como lo expresaron la mayor parte de la población está compuesta por padre, madre e hijos. El 16\% restante tiene una familia de tipo extensa donde además de padres e hijos se aúnan abuelos y suegros. Con respecto al rol que ejercen en su núcleo familiar los soldados, suboficiales y oficiales que participaron en sus respectivos grupos focales, se pudo evidenciar que el $100 \%$ lo relaciona directamente con su función como proveedores económicos, "en casa soy quien aporta lo económico y las cosas que se necesitan" (E 6). De otra parte, el 50\% se considera la cabeza visible del núcleo familiar, el $40 \%$ se concibe como educador y apoyo para sus familias, mientras que solamente el $10 \%$ se perciben como generadores de bienestar y felicidad, esto denota el establecimiento de relaciones de proveedores desde lo económico por parte de la mayoría de las cabezas de hogar, mientras que lo afectivo y relacional como son la construcción de vínculos se determina con un porcentaje muy bajo.

En relación con la dinámica familiar, el $90 \%$ manifestó que esta se encuentra determinada por la mujer (esposa, compañera o cónyuge) "mi mujer ha desempeñado un rol de papá, mamá y amiga y está más tiempo con mis hijos pues yo como militar he estado siempre un poco distante" (E 12), esto es comprensible porque debido al tipo de trabajo que los hombres desempeñan al interior de las Fuerzas Armadas los condicionan a estar ausentes por largos períodos debido a las comisiones y misiones que les son delegadas y las esposas o compañeras al convivir la mayor parte del tiempo con los hijos y demás miembros de la familia, son quienes establecen las normas de convivencia y pautas de crianza en el hogar.

Un 10\% de los participantes de los grupos focales, manifestó que sus hijos les reciben como extraños al llegar a casa y llama la atención que el $10 \%$ busca ser visto por sus hijos como amigo, debido a las circunstancias que les llevan a ausentarse por largos periodos de tiempo de casa. Con respecto a la prácticas de crianza, el $90 \%$ de la población precisa que estas prácticas son establecidas de acuerdo con las maneras en que fueron educados por sus padres, "En las prácticas de crianza que he tenido en mi casa, mi papá también fue militar, he tratado de mejorar un poco" (E.1), por tanto, repiten los mismos comportamientos, lo cual se evidencia cuando responde que sus prácticas de crianza: "van ligadas a todos los principios o algunos principios que me inculcaron a mí como hijo" (E.4). Al ser replicadores de las pautas de crianza recibidas en sus hogares ven las relaciones de poder y autoridad como algo natural siendo un parámetro diario que vivencian en sus trabajos y que se constituye en un factor desencadenante de VIF, sin que sea entendido como tal por parte de la población objeto del presente estudio.

Asimismo, el 40\% de la población asevera que las practicas van ligadas al ejemplo de vida que ellos pueden dar a sus hijos y que se encaminan a educar en valores tales como el respeto, la honestidad, la tolerancia, la lealtad, entre otros.

Al momento de indagar sobre la forma cómo se generan las pautas de crianza, el $100 \%$ de la población hace hincapié en la importancia de generar acuerdos al interior de la estructura familiar con la pareja, con la finalidad de trabajar en la formación en valores y en la forma adecuada de tratar a los hijos en lugar de acciones y comportamientos violentos, sin embargo el $80 \%$ resalta que se debe procurar una formación dónde genere el máximo respeto por la autoridad de los padres "hacer distintas formas de que el niño le capte a uno el mensaje para que realmente crezca una mejor persona" (E.7), esto evidencia que el respeto por la autoridad es prioritario para la población.

En cuanto a los valores fundamentales que se viven en familia el $100 \%$ identificó como principal al respeto, haciendo énfasis en "la persona, la dignidad y el gobierno" (E.10); seguido por la honradez y la honestidad en un 30\%, el amor por el $20 \%$ mientras que la tolerancia y la humildad el $20 \%$ y la lealtad, la confianza y la comunicación por el $10 \%$. Es importante resaltar que el $10 \%$ los relaciona con los valores tradicionales 
pertencientes a hogares nucleares" mientras que otro $10 \%$ los concibe como elementos esenciales presentes en todo actuar de la persona.

Al indagar por las formas más comunes de comunicación que se presentan entre los miembros de sus familias se presenta confusión en cuanto a las formas y los tipos de comunicación, "Las formas más comunes de comunicación que aplicó en mi hogar, básicamente siempre las hago pensando en el raciocinio" (E.11). Es por eso que, con relación a los tipos de comunicación, el 90\% manifiesta que utiliza la comunicación verbal y la forma es de tipo presencial o utilizando medios tecnológicos por la realidad de sus trabajos y el $10 \%$ insiste en escuchar y ser escuchado. Es importante anotar que hacen referencia a comunicarse de forma racional, directa, de forma básica y explicita a partir del dialogo.

\section{Concepciones de Violencia Intrafamiliar}

En relación con las concepciones de VIF, el $90 \%$ manifiesta que se trata todas aquellas agresiones físicas, verbales o psicológicas que se le pueden hacer a un integrante de su núcleo familiar afectando a la pareja o a los hijos, "Violencia intrafamiliar lo que uno escucha, lo que uno mira, lo que uno presencia, a veces violencia intrafamiliar es todas aquellas agresiones físicas psicológicas que se le pueden hacer a un integrante de nuestro núcleo familiar" (E.5). Las personas participantes del estudio ampliaron el concepto entendiendo que no solamente se trata de las agresiones sino también de los comportamientos que afectan negativamente al grupo familiar, es importante tener en cuenta que en los grupos focales se coincide en un $20 \%$ que existe la VIF con antecedentes históricos asociadas a la música u otros mensajes del medio, ya que se invita de forma directa o indirecta a solucionar los problemas recurriendo a la violencia. De otra parte, el $40 \%$ de la población amplia el concepto y le entienden como actos inhumanos, aberrantes, haciendo énfasis en la violencia física, pero ninguno la relaciona con el tipo de comportamientos y circunstancias que se viven al interior de la institución.

Un porcentaje superior al $80 \%$ de los participantes definen la VIF como diversas formas de agresión que se pueden presentar al interior de un hogar inicialmente de manera física, psicológica o verbal que hiere y lastima de múltiples formas a los integrantes de la familia dejando secuelas de tipo físico y psicológico. Adicional a ello, el $40 \%$ la define como "un acto atroz o una forma de barbarie hacia la otra persona y sus hijos" (E.10).

Es importante resaltar que también es definida como la incapacidad de expresar el inconformismo de manera pasiva, tolerante y se expresa en diversos tipos de abuso de autoridad, por parte de un miembro de la familia en repetidas ocasiones, pero hacen énfasis que quienes más la viven son la esposa e hijos. Esto evidencia un elemento de trabajo que se replica al interior de las familias, pero que para ellos no es claro que se trata de VIF sino de una forma de entender y hacer respetar la autoridad.

El 100\% de la población estudiada precisa que existen dos tipos de VIF la física y la psicológica, y solo el $60 \%$ reconoce la verbal, mientras que el 20\% reconoce otras formas de VIF como son la violencia sexual, la económica, la de raza y la violencia cultural, esto porque el $30 \%$ de la muestra son pertenecientes a otras etnias o grupos indígenas. En los grupos focales el $60 \%$ afirmaron de diversas maneras que los diferentes tipos de VIF afectan a la persona a nivel emocional y que la degradan como ser humano.

Es necesario resaltar que más del $80 \%$ de la población objeto de estudio hizo referencia a la existencia de indicadores de violencia física en sus diversas intervenciones en los grupos focales, pero cuando se les preguntó directamente si en sus hogares se vivía el fenómeno, no lo reconocen, e insisten que las relaciones se basan en el dialogo y la comunicación.

En cuanto a las condiciones que promueven la VIF el $50 \%$ manifiesta que el irrespeto, no saber escuchar y la falta de comunicación son los principales generadores de VIF y el $40 \%$ que "solo se da si hay dos personas dispuesta a ello" (E.3). Mientras que el $20 \%$ precisa que es generada por la desconfianza y elementos de orden económico, social y cultural y el $10 \%$ habla de la intolerancia y falta de amor propio. Es importante resaltar que más del 90\% de los participantes de los grupos focales enfatiza que cualquier miembro de la familia puede ser generador de VIF, no obstante, de acuerdo con la percepción de los miembros del batallón, el 50\% cree que son los hombres quienes más influyen para que este fenómeno se presente 
y llama la atención que el $20 \%$ consideran que son los hijos.

\section{DISCUSIÓN}

En concordancia con lo que plantea Pérez (2005), quien afirma que la VIF se traduce en la dinámica del marco sociocultural que impone una relación de víctima y victimario, los datos recolectados y analizados permitieron deducir que hay precisión global en el concepto de VIF en el grupo estudiado, y que esta es comprendida por los participantes como todas aquellas situaciones o formas de abuso de poder, maltrato físico o psicológico de un miembro de la familia hacia otros, las cuales se desarrollan dentro del contexto familiar y que causan algún tipo de daño en cualquiera de sus formas.

En este sentido, se puede afirmar, de acuerdo con los datos recopilados en la investigación, que las familias de origen son altamente influyentes en la manera que se desarrollan los comportamientos de cada uno de sus integrantes, siendo una consecuencia la réplica de las mismas pautas con sus hijos, Aunado a lo anterior es necesario considerar y analizar las pautas de crianza a la luz de los procesos formativos de los militares puesto que replican inconscientemente la forma de entender la autoridad como autoritarismo, puesto que esta no puede ser cuestionada, en su praxis debe ser siempre respetada sin importar otras justificaciones. Por lo anterior es necesario generar el desarrollo de habilidades y destrezas que conduzcan a adquirir competencias ligadas a la inteligencia emocional, social y moral, conducentes a disminuir la VIF al interior de las estructuras familiares y a fortalecer estrategias que fomenten la sana convivencia.

De igual forma, las respuestas permitieron evidenciar que la mayor parte de los entrevistados percibe la relación entre el ejercicio laboral y la VIF, debido a la presión del trabajo, acuñando que los procesos formativos pueden mejorar la regulación de estas emociones. Lo anterior hace referencia a las situaciones condicionadas en escenarios de relaciones de poder que trasladados al ámbito doméstico pueden generan formas de abuso al interior de la familia (Morales, 2004).

De otra parte, el separarse varios meses de la pareja y el poco tiempo para compartir con la familia puede ocasionar desapego y desconfianza, generando algunas situaciones que impacten la tranquilidad de la dinámica familiar. Otro elemento mencionado es el de asumir comportamientos similares a los desarrollados en el ambiente laboral, sin embargo, la totalidad expresa que se hace necesario evitar llevar las relaciones laborales a las familias, así como repetir pautas de crianza generadoras de situaciones que desencadenan en VIF.

Lo anterior se presenta debido a carencias en la persona como son la falta de seguridad, cariño hacia los otros, amor, educación, también por elementos como: antecedentes familiares de violencia abuelos, elección inapropiada de pareja, pretender cambiar a una persona, altivez, los estratos sociales bajos, el ambiente de violencia en algunos pueblos o barrios; porque se presentan chismes, inconformidades, surgimiento de expresiones inapropiadas y malas palabras, desacuerdos en la pareja, estrés en el trabajo, ira, celos y el alcohol entre otros.

\section{CONCLUSIONES}

A partir de la investigación realizada fue posible comprender que el grupo de militares participantes conciben la familia como la institución social primaria en la que se generan las interacciones básicas entre las personas, fuente de motivación para realizar su labor diaria. Hay claridad en el concepto de Violencia Intrafamiliar entendido como todas aquellas agresiones físicas, verbales o psicológicas que se le pueden hacer a un integrante de su núcleo familiar afectando a la pareja o a los hijos.

También entienden al VIF como la incapacidad de expresar el inconformismo de manera asertiva y tolerante, lo que se traduce en diversos tipos de abuso de autoridad por parte de un miembro de la familia en repetidas ocasiones, pero hacen énfasis que quienes más la viven son la esposa e hijos.

Los tipos de VIF que más se presentan en el grupo de estudio son la física y la psicológica y en un porcentaje más bajo la violencia cultural y la de género similar al que se vivía en las familias de las cuales previenen.

En razón a lo anterior, expresan que la VIF se produce por la incapacidad de controlar las emociones, falta de respeto y comunicación, en este orden de ideas, los comportamientos que 
viven en su relación laboral evidencian conductas con manifestaciones de agresiones físicas y verbales, situación que pueden desencadenar comportamientos violentos en el hogar sino se manejan de forma adecuada con base en los procesos formativos y respeto por los miembros de la familia.

En el batallón las familias son constituidas de forma legal o religiosa y de tipo nuclear por estar compuestas por padres, madres e hijos y en un porcentaje menor son familias construidas por el vínculo simplemente, en un menos porcentaje son familias de tipo extenso por convivir con hermanos, padres o cuñados.

Los padres se consideran como proveedores económicos en un alto porcentaje, educadores y generadores de bienestar, las relaciones de poder son establecidas desde lo económico. También, en los hogares las dinámicas familiares se encuentran determinadas por las mujeres debido a la ausencia de los padres, por su situación laboral han creado mecanismos de compensación por la ausencia prolongada del hogar y eso puede generar ausencia de normas y en último caso autoritarismo, siendo estos dos generadores de VIF.

En el marco del proceso investigativo, no se reconoce la violencia intrafamiliar en los hogares, no obstante, en las respuestas se reiteran elementos que pueden llevar a la presencia de conductas agresivas a partir del establecimiento de vínculos abusivos, lo que generalmente se componen por el desequilibrio del poder, la autoridad y jerarquía.

Asimismo, se establecen pautas de crianza de acuerdo con los comportamientos que aprendieron en sus casas y tienden a repetirlos, de igual forma el respeto es el principal valor, especialmente por la autoridad de los padres.
Los entrevistados presentan confusión a nivel de la conceptualización epistémica de los valores, las formas y tipos de comunicación, de otra parte, el separarse varios meses de la pareja y el poco tiempo para compartir con la familia puede ocasionar desapego y desconfianza, generando algunas situaciones que pueden llegar a desembocar en conductas violentas.

Las configuraciones familiares emergen de forma inconsciente debido a las pautas de crianza ya las concepciones de vida que emergen de su actividad laboral, allí se hace una reflexión clara a no querer entornos violentos en su hogar, ya que en la cotidianidad están lidiando con escenarios agrestes, esperan que su casa sea convertida en su refugio.

Se hace evidente la necesidad de establecer una comunicación asertiva y clara de los integrantes de la familia quienes tendrán que adaptarse para llegar a acuerdos o encontrar estrategias que les permitan funcionar de una manera óptima articulando las dinámicas propias de su cotidianidad. También, los participantes mencionan que los valores trabajados al interior de sus familias son los tradicionales de sus familias de origen y a la institución a la cual pertenecen, los cuales han sido enseñados y en algunas ocasiones impuestos, como parte de la repetición de las pautas de crianza en sus hogares.

En general es importante reconocer las concepciones de este grupo de militares asociadas a diversas dimensiones de su vida, hacer un llamado para que se generen estrategias cada vez más sólidas y espacios donde estos puedan desempeñarse en cada uno de sus roles. En última instancia reflexionar, actuar y propender serán palabras asociadas a un mayor bienestar propio y del núcleo familiar en una sociedad más justa, consciente y responsable socialmente.

\section{BIBLIOGRAFÍA}

- Alonso Varea, J. y Castellanos Delgado, J. (2006). Por un enfoque integral de la violencia familiar. Psychosocial Intervention, 15(3) 253 - 274. Recuperado en 27 de febrero de 2020 de https://n9.cl/5qd7l.
- Arena Borreo, D.; Herrera Vargas, D. \& Montenegro, M. (2013). Experiencia de la conyugalidad en los oficiales y suboficiales de la Fuerza Aérea colombiana - Comando Aéreo de Transporte Militar- CATAM, 
durante el año 2013 "experiencias de vida, parejas en el contexto militar". Tesis de grado. Universidad Colegio Mayor de Cundinamarca. Recuperado el 15 de marzo de 2020 de https://n9.cl/x13ho.

- Básmeson, J. y Caicedo, L. (2011). La desintegración familiar y su consecuencia en el rendimiento escolar de los estudiantes de los $4^{\circ}, 5^{\circ}$ y $6^{\circ}$ años de la escuela Fe y Alegría, Curundú, Panamá (Licenciatura). Universidad Latina de Panamá. Facultad de Ciencias de la Educación.

- Bonilla-Castro, E. y Rodríguez, P. (2015). Más allá de los dilemas de los métodos. La investigación en ciencias sociales. Bogotá: Norma.

- Carbonell, J; Carbonell, M y González Martín, N (2012). Las Familias en el siglo XXI: Una mirada desde el Derecho. Serie: Estudios Jurídicos, Núm. 205. México: UNAM, IIJ.

- Congreso de Colombia. (24 de julio de 2000). [Ley 599 de 2000]. Por la cual se expide el Código Penal. República de Colombia: Art. 229.

- Díaz Colorado, F. (2012). La justicia desde las víctimas. Revista Misión Jurídica, (5): 267 - 275. DOI: https://doi. org/10.25058/1794600X.57

- EL ESPECTADOR. (16 de diciembre de 2017). Más de 70 mil casos de violencia intrafamiliar entre enero y noviembre de 2017. Recuperado en 10 de marzo de 2020 de https://www.elespectador.com/ noticias/nacional/mas-de-70-mil-casosde-violencia-intrafamiliar-entre-enero-ynoviembre-de-2017-articulo-728844

- Espitia Carrascal, R. E., y Montes Rotela, M. (2009). Influencia de la familia en el proceso educativo de los menores del barrio Costa Azul de Sincelejo (Colombia). Investigación y desarrollo: revista del Centro de Investigaciones en Desarrollo Humano, 17 (1), 84-105. Recuperado en 21 de febrero de 2020 de https://dialnet.unirioja.es/ servlet/articulo?codigo $=3101779$.

- Garrido Chacana, C. (2013). Características de la Violencia Intrafamiliar. [En línea]. Recuperado en 21 de febrero de 2020 de http://www.carlosgarridochacana. cl/index.php/articulos/item/4 7 caracteristicas-de-la-violenciaintrafamiliar.

- González Cuétara, J. M.; Loy Vera , B. H., Viera Ponce, T., Lugo Jáuriga, B. R., Rodríguez García, C. y Carvajal Rodríguez, E. (2018). Violencia intrafamiliar. Una mirada desde la adolescencia. Acta Médica Del Centro, 273-285. Recuperado en 12 de marzo de febrero de 2020 de http://www. revactamedicacentro.sld.cu/index.php/ amc/article/view/926/1189.

- González Rincón, J., Gross Parra, K. y Pulido López, J. A. (2014). Fortalecimiento del vínculo afectivo y corresponsabilidad en padres, madres y cuidadores del nivel de sala materna y caminadores del Jardín Infantil La Paz de la Secretaria Distrital de Integración Social. Recuperado en marzo 25 de 2020 de https://n9.cl/bk9b.

- Guío Camargo, R. E. (2009). El concepto de familia en la legislación y en la jurisprudencia de la Corte Constitucional colombiana. (Universidad Católica de Colombia, Ed.) Studiositas, 4(3), 65-81. Recuperado en 4 de febrero de 2020 de https://n9.cl/9o6p.

- Guardian Fernández, A. (2007). El paradigma cualitativo en la investigación educativa. Colección IDER.

- INMLCF (Instituto Nacional de Medicina Legal y Ciencias Forenses). (2016). Datos para la vida. Forensis, 18(1), 649. [En línea]. Recuperado en 17 de febrero de 2020 de https://n9.cl/3ecs.

- -----. (2017a). Informe de Violencia Intrafamiliar Colombia. Procuest. Recuperado en 4 de marzo de 2020 de https://n9.cl/rg2c.

- -----. (2017b). Informe Lesiones Fatales y No Fatales de Causa Externa según Clasificación del Contexto de Violencia y Desaparecidos. Recuperado en 11 de marzo 
de 2020 de https://www.medicinalegal. gov.co/

- ----. (2018). Boletines estadísticos mensuales 2018. Subdirección de servicios forenses Centro de referencia nacional sobre violencia. Recuperado en noviembre 6 de 2019 de febrero de 2020 de https:// n9.cl/2qwa.

- Martínez Pacheco, A (2016). La violencia. Conceptualización y elementos para su estudio Política y Cultura. Distrito Federal, México: Universidad Autónoma Metropolitana Unidad Xochimilco. Recuperado en 2 de marzo de 2020 de https://n9.cl/htswb.

- Páez Martínez, R. M., Meza Rueda, J. et al. (2016). Familia, escuela y desarrollo humano: rutas de investigación educativa (1 ed.). Bogotá: Universidad de La Salle, CLACSO. Recuperado en 15 de febrero de 2020 de https://n9.cl/pthei.

- Ramírez Mahecha, Ortiz Quevedo \& Palacios Rozo (2018). Prácticas docentes: una aproximación a la paz. En: Acosta, W. (Ed) La innovación como agenciamiento educativo comunitario. (pp. 155-166). Colombia. Ed: Ediciones Unisalle.

- Ministerio de Salud y Protección Social. (2012). Sistema de vigilancia epidemiológica de la violencia intrafamiliar. Recuperado en 4 de febrero de 2020 de https://n9.cl/m6y2.

- Morales Gil, I. M. (2004). Violencia intrafamiliar maltrato infantil. En Mompart, M (Ed) La enfermería viva. Actualizaciones año 2014. (pp. 259-271). España: Difusión Avances de Enfermería. Recuperado en 13 de marzo de febrero de 2020 de https:// n9.cl/p9y8k.

- Navarro, K. (2013). Conflicto, Violencia y No-Violencia. Revista Misión Jurídica, 6 (6): 95-107. DOI: https://doi. org/10.25058/1794600X.61

- Ocampo Otálvaro, L. E., y Amar Amar, J. J. (enero-junio de 2011).Violencia en la pareja, las caras del fenómeno. Revista Científica Salud Uninorte, 27 (1). Recuperado en 15 de febrero de 2020 de https://n9.cl/htrjo.

- OMS (Organización Mundial de la Salud). (2002). Informe Mundial sobre Violencia y Salud. Recuperado en 08 de febrero de 2020, de https://n9.cl/vhhic.

- OMS Organización Mundial de la Salud. (2013). Informe mundial sobre la violencia y la salud. Recuperado en 08 de marzo de 2020 de https://n9.cl/khlt.

- Pérez Contreras, María de Montserrat. (2005). Aproximación a un estudio sobre vulnerabilidad y violencia familiar. Boletín mexicano de derecho comparado, 38 (113), 845-867. Recuperado en 04 de febrero de 2020, de https://n9.cl/ivxc.

- Piza, M. (2014). Estudios sobre la violencia intrafamiliar en Colombia el tratamiento desde la perspectiva de salud física y mental. Recuperado en 04 de febrero de 2020 de https://n9.cl/3v3kx.

- Ramos Rangel, Y. \& González Valdés, M. D. (2017). Un acercamiento a la función educativa de la familia. Recuperado en 06 de enero de 2020 de https://n9.cl/8n1a0.

- Rodrigo, M. J., Máiquez Chaves, M. L., Martín Quintana , J. C., \& Byme, S. (2008). Preservación familiar un enfoque positivo para la intervención con familias (1 ed.). España: Madrid: Pirámide, D.L.

- SECRETARIA DE SALUD DE BOGOTÁ D.C. (2010). Sistema de Vigilancia Epidemiológica de la Violencia Intrafamiliar, el Maltrato Infantil y la Violencia Sexual SIVIM. Recuperado en 26 de enero de 2020 de http://www.saludcapital.gov.co/

- Valle, A.D (2012). La investigación pedagógica. Otra mirada. Recuperado en 28 de enero de 2020 de https://n9.cl/132jm.

- Vila, Ignacia (2008). Familia, escuela y comunidad (1 ed.). Girona: Horsori Editorial. 